\title{
ARTICLE \\ Oxytocin receptor DNA methylation and alterations of brain volumes in maltreated children
}

\author{
Takashi X. Fujisawa ${ }^{1}$, Shota Nishitani $\mathbb{D}^{2,3}$, Shinichiro Takiguchi ${ }^{4}$, Koji Shimada ${ }^{1}$, Alicia K. Smith $\mathbb{D}^{2,3}$ and Akemi Tomoda ${ }^{1,4}$
}

\begin{abstract}
Although oxytocin (OXT) plays an important role in secure attachment formation with a primary caregiver, which is impaired in many children with childhood maltreatment $(\mathrm{CM})$, epigenetic regulation in response to $\mathrm{CM}$ is a key factor in brain development during childhood. To address this issue, we first investigated differences in salivary DNA methylation of the oxytocin receptor (OXTR) between CM and Non-CM groups of Japanese children (CM: $n=44$; Non-CM: $n=41$ ) and its impact on brain structures in subgroup analysis using brain imaging and full clinical data (CM: $n=24$; Non-CM: $n=31)$. As a result, we observed that the CM group showed higher CpG 5,6 methylation than did the Non-CM group and confirmed negative correlations of gray matter volume (GMV) in the left orbitofrontal cortex (OFC) with CpG 5,6 methylation. In addition, the CM group showed significantly lower GMV in the left OFC than did the Non-CM group. Furthermore, as a result of examining the relationship between GMV in the left OFC and psychiatric symptoms in CM, we observed a negative association with insecure attachment style and also confirmed the mediation effect of left-OFC GMV reduction on the relationship between OXTR methylation and insecure attachment style. These results suggest that any modulation of the oxytocin signaling pathway induced by OXTR hypermethylation at CpG 5,6 leads to atypical development of the left OFC, resulting in distorted attachment formation in children with CM.
\end{abstract}

Neuropsychopharmacology (2019) 44:2045-2053; https://doi.org/10.1038/s41386-019-0414-8

\section{INTRODUCTION}

Childhood maltreatment (CM) is estimated to affect up to $30 \%$ of children worldwide [1]. CM encompasses a spectrum of abusive actions (sexual, physical, or emotional abuse) or lack of actions (physical or emotional neglect) by the parent or other caregivers. $\mathrm{CM}$ reportedly increases the risk for psychiatric disorders and results in several lifelong difficulties [2]. For example, CM could distort attachment to and sense of intimacy toward significant others [3]. Therefore, many maltreated children have interpersonal communication problems that persist later in life, with negative consequences for their social relationships, including those with friends, partners, and families [4-6].

Numerous neuroimaging studies about adults with CM experiences have suggested that structural and/or functional changes are caused in various brain regions (for review [7]). Structural neuroimaging studies have provided evidence for deficits in brain volume, the gray and white matter of several regions, most prominently the prefrontal and anterior cingulate cortices [8], but also the hippocampus, amygdala, and corpus callosum [9]. Additionally, diffusion tensor imaging studies have produced evidence for deficits in structural interregional connectivity between these areas, suggesting neural-network abnormalities [10]. Similarly, functional imaging studies have supported this evidence by reporting atypical activation in the same brain regions during executive functions [11], reward processing [12], and emotional processing $[8,11]$. Although these studies have elucidated the link between $C M$ and the matured adult brain and the clinical consequences based on neurobiological evidence, it remains unclear how CM impacts brain development and function during childhood and adolescence, periods of major brain reorganization.

Childhood and adolescence are characterized by remarkable change in physical and psychological development, brain structure, and neuroendocrine function. Although the developmental process is guided by genetic factors, development is ultimately determined by life experience and the living environment. From birth, parent-infant interaction modulates fundamental brain processes [13,14], whereas more complex psychological development is driven by socializing with peers [15]. Considering this issue, several recent imaging studies have also been conducted with children and adolescents in whom limited time had passed since their CM exposure (for reviews $[16,17])$. While studies have revealed how CM modulates the developing brain, advancing the reorganization of the brain's structural/ functional networks during childhood and adolescence [18, 19] (for reviews $[16,20]$ ), the potential biological biases acting to link vulnerable brain structures and functions caused by $\mathrm{CM}$ remain undetermined.

Epigenetic modifications, such as DNA methylation, provide a critical link between external environmental factors and longlasting phenotypic change. The mechanisms supporting the role of early-life experience on DNA methylation for social brain development remain unclear, but differences in DNA methylation patterns of the stress-response genes in the brain and periphery

\footnotetext{
${ }^{1}$ Research Center for Child Mental Development, University of Fukui, Fukui, Japan; ${ }^{2}$ Gynecology and Obstetrics, Emory University School of Medicine, Atlanta, GA, USA; ${ }^{3}$ Psychiatry and Behavioral Sciences, Emory University School of Medicine, Atlanta, GA, USA and ${ }^{4}$ Department of Child and Adolescent Psychological Medicine, University of Fukui Hospital, Fukui, Japan

Correspondence: Akemi Tomoda (atomoda@u-fukui.ac.jp)

These authors contributed equally: Takashi X. Fujisawa, Shota Nishitani.
}

Received: 17 January 2019 Revised: 7 April 2019 Accepted: 26 April 2019

Published online: 9 May 2019 
in individuals who have experienced CM have been associated with psychopathology and vulnerable brain structures in adulthood [21, 22]. In addition to stress-response genes, recent studies have linked adverse social environments and DNA methylation of the oxytocin receptor (OXTR) gene to negative cognitive schemas associated with attachment style [23], internalizing problems [24], and psychiatric symptoms [25]. There is increasing evidence that OXT plays an important role in regulating social-attachment behavior in diverse species [26]. In humans, many studies have revealed that OXT modulates mother-infant bonding, affiliation, and attachment [27], and children who have experienced maltreatment and who are lacking attachment formation or bonding with a "primary caregiver" have atypical OXT secretion patterns $[28,29]$.

Here we investigated the relationship between DNA OXTR methylation, $\mathrm{CM}$, and its impact on the brain structures of children for whom limited time has passed since their CM exposure. First, we collected epigenetic data via saliva samples from children who had experienced maltreatment and investigated the crosssectional case-control comparison of OXTR methylation to locate OXTR CpG sites associated with CM exposure. Second, structural neuroimaging data were collected using magnetic resonance imaging (MRI), and we analyzed the correlation between the methylation of OXTR CpGs and gray-matter volumes (GMVs) using a voxel-based morphometry (VBM) approach. Thus, our main hypothesis was that the OXTR methylation status would be modified by the experience of CM and impact brain structures implicated in social behavior via atypical brain development during childhood and adolescence.

\section{METHODS}

\section{Ethics statement}

The study protocol was approved by the Ethics Committees of the University of Fukui, Japan (Assurance no. FU23-43), and the study was conducted in accordance with the Declaration of Helsinki and the Ethical Guidelines for Clinical Studies of the Ministry of Health, Labour and Welfare of Japan. All children and a parent or director of a child welfare facility provided written informed assent and consent, respectively, for participation in this study.

\section{Participants}

Eighty-five Japanese children and adolescents aged 6-20 years (55 boys, 30 girls; mean age \pm SD: $12.9 \pm 2.6$ years) participated in this study. The maltreated group consisted of 44 children with maltreatment experiences (CM group) who were recruited from the University of Fukui Hospital and the local child welfare facilities in Fukui Prefecture, Japan. All the children had experienced physical, emotional, sexual abuse, and/or neglect early in life prior to coming into care. Although the children were not living with their biological parents, they lived in a stable environment in a child welfare facility. The non-maltreated group consisted of 41 children with no history of maltreatment (Non-CM group), who were recruited from the local community via advertisements and served as controls.

For a subset of 55 (CM, 24; Non-CM, 31) participants, two licensed pediatric psychology clinicians conducted brain imaging and detailed clinical assessments to investigate the neural correlates associated with CM. To exclude other psychiatric diseases (e.g., mood-related disorders, anxiety disorders and stress disorders) and neurodevelopmental disorders (autism spectrum disorder and attention-deficit hyperactivity disorder), this subset of participants was administered the Mini-International Neuropsychiatric Interview for Children and Adolescents [30]. Participants who had a full-scale intelligence quotient (FSIQ) $<70$ on the Wechsler Intelligence Scale for Children-Fourth Edition or the Wechsler Adult Intelligence Scale-Third Edition were excluded $[31,32]$. Participants were also excluded if they had history of substance abuse, head trauma with loss of consciousness, marked fetal exposure to alcohol or drugs, perinatal or neonatal complications, neurological disorders, sleep disturbances, or medical conditions that might adversely affect growth and development. Most participants with CM $(80.0 \%)$ were medication naïve, except for four (20.0\%) who were using methylphenidate but had a wash-out of $72 \mathrm{~h}$ before scanning.

In addition to the clinical assessments, we evaluated the participants' psychiatric symptoms or psychological status using the following four scales under the supervision of pediatric psychology clinicians. The Child Abuse and Trauma Scale (CATS) was administered to assess the participants' maltreatment in early childhood [33]. The Depression Self-Rating Scale for Children (DSRSC) was used to measure depressive symptoms [34]. Behavioral/emotional problems were assessed using the Child Behavior Checklist (CBCL) [35]. To assess the secure or insecure (avoidant and ambivalent) attachment style of the participants, they completed the Japanese version of the Attachment Style Measures revised to a multiple-point Likert-type scale (Internal Working Model Scale; IWMS) [36-38] that has confirmed reliability and validity in both the original $[36,37]$ and the Japanese $[38,39]$ versions and is widely used for measuring attachment style.

\section{Saliva collection and DNA extraction}

Saliva samples were collected using Oragene Discover OGR-500 kits (DNA Genotek Inc. Kanata, ON). DNA was extracted using prep $T^{\oplus} \cdot \mathrm{L} 2 \mathrm{P}$ reagent (DNA Genotek) and was quantified with PicoGreen (Quant-iT ${ }^{\top \mathrm{M}}$ PicoGreen ${ }^{\circledast}$ dsDNA Assay Kit, Thermo Fisher Scientific Inc., Waltham, MA).

OXTR DNA methylation

One $\mu \mathrm{g}$ of DNA was treated with sodium bisulfite using the EpiTect Fast Bisulfite Kit (Qiagen, Hilden, Germany). The OXTR gene (chr3: 8792095 to 8811300 ; GRCh37/hg19 build) was interrogated using EpiTYPER (MassARRAY system; Agena Bioscience, San Diego, CA) according to the manufacturer's instructions. We aimed to target a specific $406 \mathrm{bp}$ region (chr3: 8 810 719-8 811 124) termed MT2 [40] located in a CpG island as shown in supplementry Fig. S1, as it is a candidate region for identifying the association of the epigenetic vulnerability for CM $[24,25]$. We selected the EpiTYPER platform to cover almost all CpG sites (20/27 CpGs in MT2) in this region by a single measurement, which is difficult by other conventional methods excluding amplicon bisulfite next-generation sequencing [41]. EpiTYPER provides an accurate and reproducible targeted quantification of individual CpGs and is based on in vitro transcription and uracil-specific cleavage of bisulfite-converted genomic DNA and matrix-assisted laser desorption/ionization time-of-flight mass spectrometry [42]. The detection precision allows quantifying subtle methylation differences down to $5 \%$. The primer set (Forward: 5'- aggaagagagGAGGTITAGTGAGAGATITAGTIAAG-3'; Reverse: $5^{\prime}$ - cagtaatacgactcactatagggagaagg ctTCCCTACTAAAAAAACCCCTACCTC-3') was designed for EpiTYPER using EpiDesigner (Agena Bioscience), and the spectrum characteristics were validated with RSeqMeth [43]. One $\mu \mathrm{L}$ of bisulfite-converted DNA was used in a $5-\mu \mathrm{L}$ reaction mixture containing $0.4 \mathrm{U}$ of PCR enzyme, 1 xPCR Buffer with $20 \mathrm{mM} \mathrm{MgCl}_{2}$, $200 \mu \mathrm{M}$ dNTPs (Agena Bioscience), and 2 pmol of each primer. Cycling conditions were: denaturation $\left(94^{\circ} \mathrm{C}\right.$ for $15 \mathrm{~min}$ ) followed by 50 cycles of amplification $\left(94^{\circ} \mathrm{C}\right.$ for $30 \mathrm{~s}, 58^{\circ} \mathrm{C}$ for $60 \mathrm{~s}$, and $72{ }^{\circ} \mathrm{C}$ for $30 \mathrm{~s}$ ) and a final extension step $\left(72^{\circ} \mathrm{C}\right.$ for $\left.10 \mathrm{~min}\right)$ (GeneAmp ${ }^{\circledR}$ PCR System 9700, Thermo Fisher Scientific). Samples were electrophoresed using $2 \%$ agarose gel to confirm amplification. The mass spectra methylation ratios were generated using EpiTYPER ver. 1.2 (Agena Bioscience). For each participant, the OXTR methylation ratios were retained and used as the criterion variable in subsequent statistical analyses. The reproducibility and sensitivity of the OXTR EpiTYPER assay product were assessed 
using commercially-available standards (EpiTect control DNA, Qiagen) run in triplicate and measured as previously shown [41].

Brain-image acquisition and preprocessing

Image acquisition was performed using a 3-Tesla scanner (Discovery MR 750; General Electric Medical Systems, Milwaukee, WI) with a 32-channel head coil. A T1-weighted anatomical dataset was obtained from each subject by a fast-spoiled gradient recalled imaging sequence (voxel size $1 \times 1 \times 1 \mathrm{~mm}, \mathrm{TE}=1.996 \mathrm{~ms}, \mathrm{TR}=$ $6.38 \mathrm{~ms}, \mathrm{TI}=600 \mathrm{~ms}$, flip angle $=11^{\circ}$, total scan time $=4$ min $50 \mathrm{~s}$ ).

VBM was performed using the Statistical Parametric Mapping version 12 (SPM12) software (Wellcome Department of Imaging Neuroscience, University College London, London, UK; http:// www.fil.ion.ucl.ac.uk/spm/software/spm12/) implemented in MATLAB 9.0 (Math Works Inc., Natick, MA). The T1-weighted images were segmented coarsely into GM, white matter, cerebrospinal fluid, and skull/scalp compartments using tissue probability maps. The Diffeomorphic Anatomical Registration through Exponentiated Lie Algebra algorithm was applied to the segmented brain tissues to generate a study-specific template and to achieve an accurate inter-subject registration with improved realignment of smaller inner structures. The segmented GM images were spatially normalized, written out with an isotropic voxel resolution of $1.5 \mathrm{~mm}$. Any volume change induced by normalization was adjusted via a modulation algorithm. Spatiallynormalized GM images were smoothed by a Gaussian kernel of 8 $\mathrm{mm}$ full width at half maximum.

\section{Statistical analysis}

Demographics and clinical characteristics were compared in the full set and the subset of participants using chi-squared and $t$ tests.

To investigate regionally-specific correlates of GMV associated with CpG 5,6 methylation, we performed multiple regression using SPM. Age, FSIQ, and total GMV were included as covariates in the model. Additionally, the DSRSC score was included as a covariate because depressive symptoms may influence regional GMV alterations [44]. Given our a priori hypotheses, we constrained our search to a predefined mask as a region of interest (ROI), which consisted of 21826 voxels. We set two ROls bilaterally, the orbitofrontal cortex (OFC) and dorsal striatum, for the following reasons. First, we previously found a positive association between brain activation in these two regions and intranasal OXT administration for maltreated children [45]. Second, previous studies have reported the expression of OXTR in these regions in humans [46]. These ROIs were defined using automated anatomical labeling as implemented in the WFU Pick-Atlas toolbox [47]. The resulting set of voxel values used for comparison generated a statistical parametric map of $t$-statistic, SPM $\{t\}$ that was transformed to a unit normal distribution (SPM $\{Z\})$. The statistical threshold was set at $P<0.001$ at the voxel level and $P<$ 0.05 with a family-wise error (FWE) correction for multiple comparisons. The anatomical localization of significant clusters was investigated with the Automated Anatomical Labeling and Broadman area atlases implemented in the TalairachClient software package. Regional GMV was compared using $t$-tests when the correlation analysis was significant. All statistical analyses were performed with SPSS Version 22 (IBM Corp., Armonk, NY) and SPM 12.

\section{RESULTS}

OXTR methylation between the groups

To evaluate the association between CM and OXTR methylation in the full set of participants $(n=85$; Table 1$)$, we compared the methylation of each CpG unit in the CM versus Non-CM groups using $t$-tests (Table 2). As a result, we observed that the CM group had significantly higher methylation at CpG 5,6 than did the Non-
CM group ( $P_{\text {corr }}<0.05$; Supplementary Fig. S2). Next, to consider the potential influence of sex and age on this finding, we also performed multiple regression. The analysis showed that 'group' was associated with CpG 5,6 methylation $(\beta=0.44, t=3.66$, $\left.P_{\text {corr }}<0.05\right)$, but sex $(\beta=0.13, t=1.28, P=0.21)$ and age were not $(\beta=0.13, t=1.08, P=0.28)$ (Table 2$)$.

Demographics, clinical assessments, and psychiatric symptoms of participant subset

To investigate the neural correlates associated with CM and OXTR methylation, we conducted brain imaging and several clinical assessments for a subset of participants $(n=55)$. The demographics, clinical assessments, and psychiatric symptoms of each group are listed in Table 3. The two groups were matched for sex. Compared to the Non-CM group, the CM group had lower age $(t[53]=3.06, P<0.001)$ and FSIQ $(t[53]=7.25, P<0.001)$ and significantly higher levels of perceived severity of maltreatment during childhood (CATS: $t[47]=5.34, P<0.001$ ). Similarly, the CM group had higher levels of psychiatric symptoms: depressive symptoms (DSRSC: $t[53]=4.45, P<0.001$ ) and total behavior problems (CBCL: $t[53]=5.56, P<0.001)$. Regarding the attachment styles as assessed by the IWMS, the CM group showed significantly lower secure style and higher insecure style than did the Non-CM group $(t[51]=2.55, P=0.014 ; t[51]=3.19, P=0.002$; respectively). There was a significant difference in total GMV between the groups. The CM group showed a $5.5 \%$ decrease in total GMV compared to the Non-CM group $(t[53]=2.30, P=$ 0.025).

Replication for CpG 5,6 methylation difference in the participant subset

The CM group had higher, although not significant, CpG 5,6 methylation than did the Non-CM group $(t[53]=1.88, P=0.066$; Table 3). We also performed a regression analysis with CpG 5,6 methylation as the dependent variable and age, FSIQ, and group (CM vs Non-CM) as independent variables. The analysis showed that the model was significant $(F[4,49]=2.56, P=0.049)$ and that 'group' was a significant predictor of CpG 5,6 methylation $(\beta=0.65, t=3.18, P=0.003)$, while age, $\mathrm{FSIQ}$, and DSRSC were not $(\beta=0.10, t=0.61, P=0.547 ; \beta=0.31, t=1.59, P=0.119$; $\beta=-0.27, t=-1.63, P=0.109$ ).

Correlation between regional GMV alterations and DNA methylation of OXTR

As shown in Fig. 1, the results of the multiple regression showed that the degree of CpG 5,6 methylation was negatively correlated with the regional GMV in the left OFC after correcting for age, FSIQ, DSRSC, and total GMV (Broadman area 11; Talairach coordinates $x=-25, y=49, z=-8, Z=4.23$; cluster size $=129$ voxels, $P=0.038$, FWE-corrected for cluster level), whereas no significant association was found in the dorsal striatum $(P>0.10)$. These negative correlations between regional GMV and OXTR methylation were significantly retained in both the CM and NonCM groups $(r=-0.45, P=0.028 ; r=-0.55, P<0.001)$. There was significant left-OFC GMV reduction in the CM group $(t[53]=2.21$, $P=0.034$; Supplementry Fig. S3).

Correlation between regional GMV alterations and clinical assessments

As described in the previous section and presented in Fig. 2a, we observed significant negative correlations between regional GMV and OXTR methylation in the CM group. We then performed correlation analysis for the CM group to confirm the relationship between left-OFC GMV reduction and clinical psychological status. The analysis showed that the insecure attachment style score was significantly correlated with the OFC GMV estimates (IWMSinsecure: $r=-0.60, P=0.003$; Fig. $2 b$ ). There were no significant correlations between the OFC GMV estimates and intellectual 
Table 1. Demographics of all participants

\begin{tabular}{|c|c|c|c|c|}
\hline & $\mathrm{CM}(n=44)$ & Non-CM $(n=41)$ & Statistics & $P$ value \\
\hline Age (years), Mean $(S D)$ & $11.4(2.90)$ & $14.5(2.23)$ & $t(83)=5.50$ & $<0.001$ \\
\hline
\end{tabular}

Table 2. Statistical analysis results using $t$-test and multiple regression analysis for each $\mathrm{CpG}$ fragment

\begin{tabular}{|c|c|c|c|c|c|c|}
\hline & MAPINFO & b.p. ${ }^{a}$ & $t$ test & $P$ value & Multiple regression analysis ${ }^{\mathrm{b}}$ & $P$ value \\
\hline CpG 1 & 8811028 & -1154 & $t(83)=1.99$ & 0.05 & $t(83)=1.82$ & 0.07 \\
\hline CpG 2,3 & 8811010,8811005 & $-1136,-1131$ & $t(83)=1.52$ & 0.13 & $t(83)=1.91$ & 0.06 \\
\hline CpG 5,6 & 8810995,8810993 & $-1121,-1119$ & $t(83)=3.56$ & $<0.001^{c}$ & $t(83)=3.66$ & $<0.001^{\mathrm{c}}$ \\
\hline CpG 7 & 8810981 & -1107 & $t(83)=1.93$ & 0.06 & $t(83)=1.61$ & 0.11 \\
\hline CpG 11,12 & 8810936,8810930 & $-1062,-1056$ & $t(83)=1.33$ & 0.19 & $t(83)=1.31$ & 0.19 \\
\hline CpG 13 & 8810924 & -1050 & $t(83)=0.53$ & 0.60 & $t(83)=0.72$ & 0.47 \\
\hline CpG 14 & 8810890 & -1016 & $t(81)=0.04$ & 0.97 & $t(81)=0.28$ & 0.78 \\
\hline CpG 15 & 8810875 & -1001 & $t(83)=0.72$ & 0.48 & $t(83)=0.88$ & 0.38 \\
\hline CpG 16 & 8810863 & -989 & $t(83)=1.60$ & 0.11 & $t(83)=2.29$ & 0.02 \\
\hline CpG 21 & 8810775 & -901 & $t(83)=0.26$ & 0.79 & $t(83)=1.13$ & 0.26 \\
\hline CpG 23 & 8810709 & -835 & $t(83)=0.23$ & 0.82 & $t(83)=1.26$ & 0.21 \\
\hline CpG 24 & 8810700 & -826 & $t(83)=1.11$ & 0.27 & $t(83)=0.72$ & 0.48 \\
\hline CpG 25,26 & 8810682,8810680 & $-808,-806$ & $t(83)=0.02$ & 0.99 & $t(83)=0.45$ & 0.66 \\
\hline CpG 27 & 8810648 & -774 & $t(83)=0.69$ & 0.49 & $t(83)=0.10$ & 0.93 \\
\hline
\end{tabular}

ability (FSIQ: $r=-0.17, P=0.416)$, severity of maltreatment (CATS: $r=-0.14, P=0.535)$, depressive symptoms (DSRSC: $r=0.08, P=$ $0.714)$, behavioral problems (CBCL: $r=0.18, P=0.404)$, and secure attachment style score (IWMS-secure: $r=-0.31, P=0.160$ ).

Furthermore, we conducted an additional path analysis to examine whether left-OFC GMV reduction mediated the relationship between OXTR methylation and insecure attachment style. As shown in Fig. 2c, OXTR methylation at CpG 5,6 was a significant predictor of the OFC GMV estimates $(\beta=-0.45, t=2.40, P=$ 0.016). Furthermore, when the OFC GMV estimates and OXTR methylation were entered simultaneously as predictors of insecure attachment style, the OFC GMV estimates were found to be significant predictors $(\beta=-0.68, t=3.66, P<0.001)$, while OXTR methylation was not $(\beta=-0.05, t=0.29, P=0.772)$. The overall model fit the data well $\left(X^{2}[1]=0.090, P=0.765, \mathrm{NFI}=0.994, \mathrm{CFI}=\right.$ 1.000 , RMSEA $<0.001, \mathrm{AIC}=16.090$ ), and then the indirect effect of OXTR methylation at CpG 5,6 on the insecure attachment style mediating left-OFC GMV reduction was confirmed to be significant by the Sobel test $(\beta=0.29, Z=2.05, P=0.040)$. Thus, these findings suggest that left-OFC GMV reduction mediates the relationship between OXTR methylation at CpG 5,6 and insecure attachment style.

\section{DISCUSSION}

In this study, we investigated the relationship between OXTR methylation, $\mathrm{CM}$, and its impact on the brain structures of children. To address this issue, after confirming that the CM group showed higher OXTR CpG 5,6 methylation than did the Non-CM group, we investigated the relationship between CpG 5,6 methylation and alterations in brain morphology using a VBM approach. We observed negative correlations of GMV in the left OFC with CpG 5,6 methylation. Additionally, there was significant left-OFC GMV reduction in the CM group. Finally, as a result of examining the relationship between GMV in the left OFC and clinical psychological status in the CM group, we confirmed a negative association with insecure attachment style and a mediation effect of left-OFC GMV reduction on the relationship between OXTR methylation and insecure attachment style. These results suggest that any modulation of the oxytocin signaling pathway induced by hypermethylation of OXTR at CpG 5,6 leads to atypical development of the left OFC, resulting in distorted attachment formation in children with $\mathrm{CM}$.

Although the role of OXTR methylation in the relationship between CM clinical phenotypes and structural brain alterations has not been extensively studied, a recent study showed that 
Table 3. Demographic and clinical characteristics for the subset of participants

\begin{tabular}{|c|c|c|c|c|}
\hline & $\mathrm{CM}(n=24)$ & Non-CM $(n=31)$ & Statistics & $P$ value \\
\hline Age (years), Mean (SD) & $12.6(2.2)$ & $14.9(2.2)$ & $t(53)=3.74$ & $<0.001$ \\
\hline Physical abuse & $16(66.7)$ & - & & \\
\hline Emotional abuse & $18(75.0)$ & - & & \\
\hline Number of types of maltreatment, Mean (SD) & $2.4(1.0)$ & - & & \\
\hline Duration (years) of maltreatment, Mean (SD) & $8.0(3.9)$ & - & & \\
\hline Duration (years) elapsed from maltreatment, Mean $(S D)$ & $5.1(4.9)$ & - & & \\
\hline WISC-IV FSIQ, Mean (SD) & $88.6(10.9)$ & $108.5(9.5)$ & $t(53)=7.25$ & $<0.001$ \\
\hline CATS total ${ }^{\mathrm{a}}$, Mean $(S D)$ & $35.0(20.9)$ & $10.9(7.0)$ & $t(48)=5.71$ & $<0.001$ \\
\hline Total GMV, Mean (SD) & $791.2(75.8)$ & $834.9(64.8)$ & $t(53)=2.30$ & $=0.025$ \\
\hline CpG 5,6 methylation, Mean (SE) & $0.07(0.03)$ & $0.05(0.02)$ & $t(53)=1.88$ & $=0.066$ \\
\hline \multicolumn{5}{|c|}{$\begin{array}{l}\text { CM children with exposure to maltreatment, Non-CM children with no history of maltreatment, WISC-IV Wechsler intelligence scale for children ver. IV, FSIQ full } \\
\text { scale I.Q., CATS child abuse and trauma scale (Sanders B et al., 1995), DSRSC Depression Self-Rating Scale for Children (Birleson P, 1981), CBCL child behavior } \\
\text { checklist (Achenbach TM \& Rescorla LA, 2001), IWMS Internal Working Models Scale (Takuma \& Toda, 1988) } \\
\text { Some subjects' data were not available due to missing values in specific questionnaires } \\
{ }^{\text {a} C h i l d ~ A b u s e ~ a n d ~ T r a u m a ~ S c a l e ~(C M: ~} n=1, \text { Non-CM: } n=3 \text { ) } \\
\text { bInternal Working Models Scale (CM: } n=2 \text { ) }\end{array}$} \\
\hline
\end{tabular}

hypermethylation of the OXTR gene is associated with smaller OFC volumes and social-cognition deficits in patients with psychotic disorders [48]. This is in line with the notion that CM increases the risk for impaired social functioning, and the CM group in our study also showed significantly reduced socio-emotional adaptaion (as shown by the CBCL or IWMS scores in Table 3). This finding is also largely consistent with the results of earlier studies that found that OXT gene methylation is negatively related to GMV reduction in brain regions related to human sociability, although the areas where the reduction was observed were not consistent [49]. This study showed for the first time that OXTR hypermethylation in children for whom limited time has passed since their CM exposure is associated with difficulties in interpersonal relationships by mediating the structural alteration on the atypical brain development.

Many studies have reported associations between exposure to early-life adversities and OXTR DNA methylation. Unternaehrer et al. reported an association between low maternal care and OXTR DNA methylation [50]. Similarly, Smearman et al. found that early abuse exposure was associated with greater methylation in two CpG sites within the OXTR promoter [25]. Additionally, Needham et al. also reported that low childhood socioeconomic status was associated with OXTR methylation [51]. The results of this study are in line with these findings on the relationships between OXTR DNA methylation and childhood adversity, as in CM. Conversely, it should also be noted that the specific CpG sites varied across studies. The results of past studies are not directly comparable with our findings because the previous studies investigated different types and timing of early-life adversities. Due to dense CpG arrangement in our targeted region, it is difficult to measure the entire region using other conventional methods. Likewise, the microarray chip covers only 1 (Illumina methylationEPIC array) or 2 (Illumina $450 \mathrm{~K}$ array) per $26 \mathrm{CpGs}$ $[23,25]$. However, some studies have focused on CpG-934 (corresponding to CpG 19 in our assay) by pyrosequencing $[24,46,52]$, but single-site analysis would not be sufficiently informative to fully characterize the influence of the region. This highlights the importance of using a similar set of CpG dinucleotides in future research to facilitate comparison across studies.

The results of the structural MRI analysis revealed an association between OXTR DNA methylation and left-OFC GMV reduction in the CM group. In this study, although we could not clarify the role of the physiological mechanism of OXTR DNA methylation in the modulation of the OXT signaling pathway, OXTR DNA methylation was found to lead to decreased OXTR expression in both animal and human studies $[53,54]$. Based on these findings, hypermethylation of CpG 5,6 may have led to the modulation of OXT signaling based on lower OXTR expression in maltreated children. It is also an interesting question how this OXTR DNA methylation selectively affects left-OFC GMV reduction because OXTR is reportedly expressed in many brain regions $[55,56]$. Although it has been suggested that coordination between the OFC and subcortical structures, such as the striatum or amygdala, via OXT has an important role in attachment formation $[57,58]$, previous research and our supplementary observation (Supplementry Fig. S4) has suggested that OXTR is relatively more highly expressed within these brain regions in animals [59] and humans $[46,60]$. Moreover, the OFC has been projected from dopaminergic neurons expressing OXTR in the ventral tegmental area [61], and the mesocortical reward pathway has also been regarded as significant in attachment formation [62]. Combined, one of the possible reasons why the relevance to OXTR DNA methylation was specifically observed in the OFC may be that the OFC is a key region of the network involved in attachment formation via OXT. This speculation is also supported by the fact that OXT administration is associated with increased OFC activity when maltreated children engage in reward tasks and with 

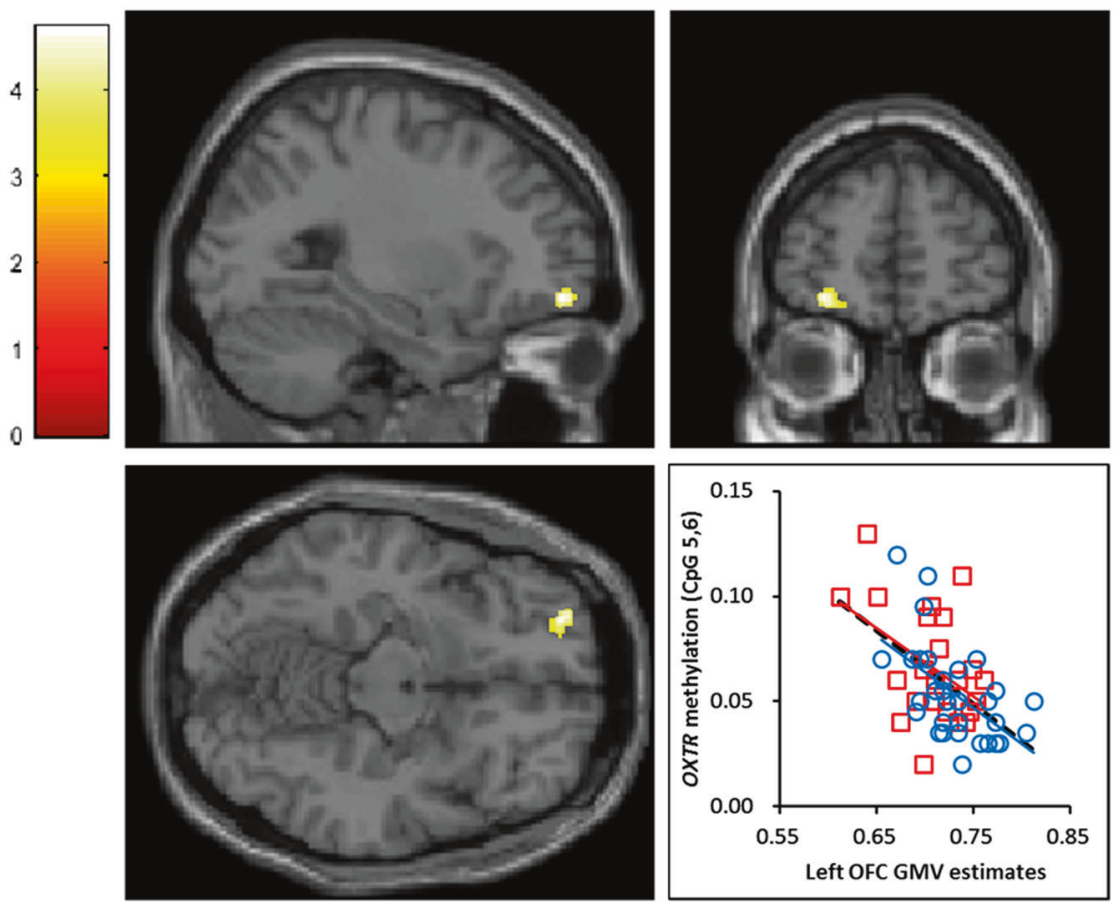

Fig. 1 Results of the multiple regression analysis between the regional gray-matter volume (GMV) and CpG 5,6 methylation. Brain region showing negative correlations between the degree of CpG 5,6 methylation and GMV in the left orbitofrontal cortex ( $-25,49,-8$ [BA 11]) as determined by multiple regression analysis. The statistical threshold for the contrasts was voxel-level $P<0.001$ uncorrected for height and cluster-level $P<0.05$ family-wise error corrected for multiple comparisons. The color bar denotes the $t$-statistic range. The scatter plot and dashed line show the association between the degree of CpG 5,6 methylation and the GMV in the left orbitofrontal cortex. The red squares and line represent the childhood maltreatment (CM) group, and the blue circles and line represent the Non-CM group. OXTR: oxytocin receptor gene, OFC: orbitofrontal cortex

improvement in their related clinical symptoms [45], and higher levels of OXTR methylation were associated with decreased functional coupling of the amygdala with the OFC involved in affect appraisal and emotion regulation [52].

Although we found a negative association between the left-OFC GMV and insecure attachment style in the CM group in this study, no previous studies have reported similar relevance of reduced GMV in the left OFC. Meanwhile, several studies have suggested that there is a positive association between insecure attachment style and emotion-regulation deficits due to functional reduction in the OFC $[63,64]$ (for review [57]). In a functional MRI study with adults, left-OFC activation while thinking negative thoughts for intimate relationship, such as with partners, was negatively correlated with insecure attachment style, suggesting that individuals with insecure style are less able to downregulate emotions evoked by negative thoughts on their relationships due to under-recruitment of the OFC [63]. Similarly, another functional MRI study with adolescents also suggested that there are negative associations between insecure attachment and brain activity during social-feedback processing of emotional faces in the left ventrolateral prefrontal cortex including the OFC (Broadman area $11)$, suggesting that adolescents with insecure attachment style are less able to perform social-feedback processing due to underrecruitment of this brain area [64]. Thus, our results on the negative association between the left-OFC GMV and insecure attachment style are in line with these findings because the reduction can be considered to lead to decrease in recruitment of resources for socio-emotional processing, and such decreased function with volume reduction may contribute to the formation of unstable attachment in individuals who have experienced CM. Furthermore, we confirmed the mediation effect of left-OFC GMV reduction on the relationship between OXTR methylation and insecure attachment style in CM and no direct relationship was found between the two. Taken together, these findings suggest that higher OXTR DNA methylation indirectly affects distorted attachment formation, i.e., insecure style, mediated by modulation of atypical development in the left OFC, one of the distributed brain regions with relatively high OXTR density.

Several limitations of this study should be noted. First, this study included a relatively small sample size and had a cross-sectional design that precluded the identification of causal links between OXTR methylation, CM, and its impact on the brain structures of children. It was also difficult to consider the difference in impact depending on the type of CM. Longitudinal studies with larger sample sizes are required to more fully elucidate these associations. Second, we adopted ROI analysis to identify structural brain alterations related to OXTR methylation. It is important to consider that a ROI approach on previously-defined regions can, however, promote biased and inappropriately constrained anatomy characterization [65]. Third, we assayed peripherally-derived DNA from saliva as a marker. A recent study showed that methylation of DNA extracted from saliva more greatly resembles the methylation patterns observed in brain tissues than those observed in the blood [66]. Thus, saliva may be a good proxy tissue for methylation studies of brain-based traits, such as sociability [49]. A public web tool is available to examine the correlation between brain, blood, saliva, and buccal cells collected from the same living human [67]. However, there is no matched probe corresponding to CpG 5, 6 in the Illumina methylationEPIC array this web tool uses, but a neighboring site (CpG 3) is included (cg17285225). Saliva methylation of this site is not correlated with brain methylation (Spearman's $\rho=0.12, P=0.7$ ), but buccal-cell methylation tends to be positively correlated with brain methylation (Spearman's $\rho=0.5, P=0.085$ ). Considering that the mean proportion of buccal epithelial cells assessed by microscopic quantitation in children (70.3\%) is significantly higher than that in 

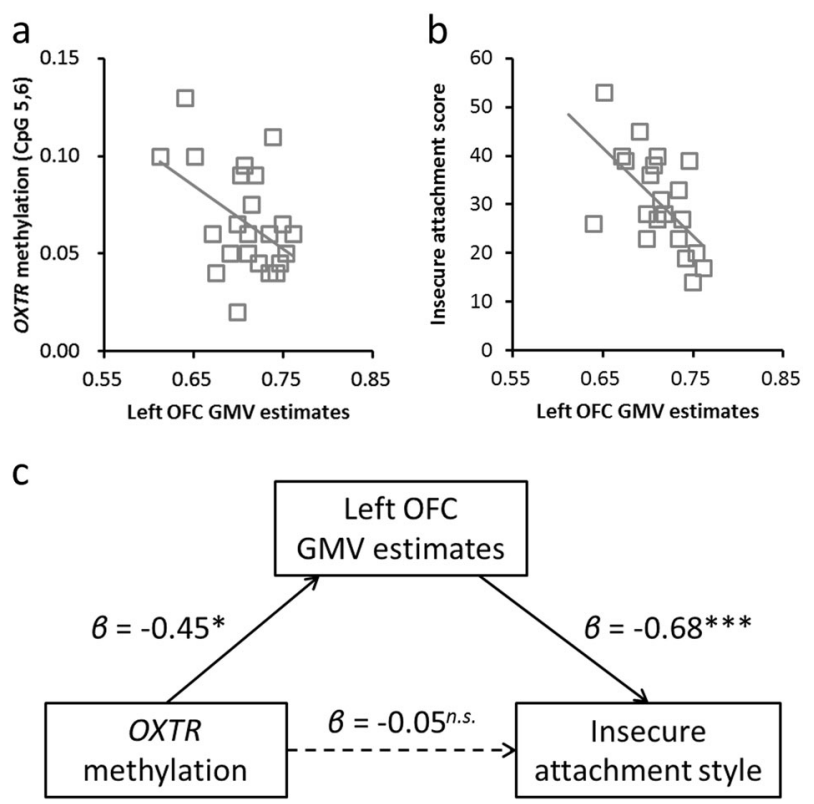

Fig. 2 The mediating role of gray-matter volume (GMV) in the relationship between oxytocin receptor gene (OXTR) methylation and insecure attachment style in childhood maltreatment. a Relationship between GMV and OXTR methylation. The vertical axis indicates OXTR methylation levels at CpG 5,6, whereas the horizontal axis indicates GMV estimates in the left orbitofrontal cortex (OFC). b Relationship between GMV and insecure attachment style. The vertical axis indicates the insecure attachment style score, whereas the horizontal axis indicates GMV estimates in the left OFC. c Path model on the mediation effect of GMV on the relationship between OXTR methylation and insecure attachment style $\left({ }^{*} P<0.05\right.$; $* * * P<0.001)$

adults (47.3\%) [68], buccal cells could comprise most of the saliva DNA in this study. From this speculation, saliva methylation in our samples may correlate with brain methylation. However, cellular heterogeneity in saliva DNA still can act as a confounding factor. Collecting buccal samples (mean proportion of buccal epithelial cells: $90.3 \%$; between-subject variation expressed as interquartile range: $10.3 \%$ ) instead of saliva to minimize cellular heterogeneity variability or microscopic quantitation of buccal cells using fresh saliva specimens for later adjusting process was required to correct for the potential confounding effect [68]. Fourth, our focus on OXTR was hypothesis-driven, and we did not conduct genomewide analysis in an exploratory manner. Moreover, although we focused on a target region called MT2 [40], one of the most interesting regions for identifying the association of the vulnerability of social brain functions, DNA methylation other than that of OXTR could also be involved and cannot be assessed using common microarray approaches. Finally, as seen in Table 1, there were substantial IQ differences in our study, with the CM group showing a lower FSIQ, as previous CM studies have also reported $[69,70]$. However, we used statistical methods to control for these confounders and adjusted for the FSIQ in the VBM analysis. Despite these limitations, this study sheds light on the neural underpinnings of social and emotional dysregulation caused by an environmental factor, i.e., the adverse experiences of children with $\mathrm{CM}$.

In conclusion, this study found significant hypermethylation of OXTR CpG 5,6 in the CM group and a correlation with GMV in the left OFC, along with reduced volume in the CM group. This GMV decrease, which is inferred to be within a region highly relevant to emotion regulation, might explain the presence of poor social functioning or emotional processing in individuals who have experienced CM. Given the important role of both the oxytocinergic system and environmental input from caregivers for attachment formation, our finding of OXTR methylation and its impact on brain structure might provide an important link for elucidating the attachment formation and clinical dysfunctions caused by its failure.

\section{FUNDING AND DISCLOSURE}

This work was supported by a Grant-in-Aid for "Creating a Safe and Secure Living Environment in the Changing Public and Private Spheres" from the Japan Science and Technology Corporation (JST)/Research Institute of Science and Technology for Society (RISTEX). This study was also partially supported by Scientific Research (B) (\#15H03106 to AT), Challenging Exploratory Research (\#15K12720 to AT), Scientific Research (C) (\#15K01753 to TXF), and Young Scientists (B) (\#15K21026 to ST) from the Japan Society for the Promotion of Science, and research grants from the Takeda Science Foundation (to AT). This study was also supported by Grant-in-Aid for Scientific Research from Japan-United States Brain Research Cooperation Program (to AT). The funders had no role in the study design, data collection and analysis, decision to publish, or preparation of the manuscript. The authors declare no conflicts of interest associated with this manuscript.

\section{ADDITIONAL INFORMATION}

Supplementary Information accompanies this paper at (https://doi.org/10.1038/ s41386-019-0414-8).

Publisher's note: Springer Nature remains neutral with regard to jurisdictional claims in published maps and institutional affiliations.

\section{REFERENCES}

1. World Health Organization. Global status report on violence prevention 2014 Geneva: World Health Organization; 2014

2. Edwards VJ, Holden GW, Felitti VJ, Anda RF. Relationship between multiple forms of childhood maltreatment and adult mental health in community respondents: results from the adverse childhood experiences study. Am J Psychiatry. 2013;160:1453-60.

3. Crittenden PM, Ainsworth MDS. Child maltreatment and attachment theory. In: Cicchetti D, Carlson V, editors. Child maltreatment: Theory and research on the causes and consequences of child abuse and neglect. New York: Cambridge University Press; 1989. p 432-63.

4. Messman-Moore TL, Coates AA. The impact of childhood psychological abuse on adult interpersonal conflict: the role of early maladaptive schemas and patterns of interpersonal behavior. J Emot Abus. 2007;7:75-92.

5. Fergusson DM, McLeod GF, John Horwood L. Parental separation/divorce in childhood and partnership outcomes at age 30. J Child Psychol Psychiatry. 2014;55:352-60.

6. Lamoureux BE, Palmieri PA, Jackson AP, Hobfoll SE. Child sexual abuse and adulthood-interpersonal outcomes: examining pathways for intervention. Psychol. Trauma . 2012;4:605-13.

7. Teicher MH, Samson JA, Anderson CM, Ohashi K. The effects of childhood maltreatment on brain structure, function and connectivity. Nat Rev Neurosci. 2016;17:652-66.

8. Dannlowski U, Stuhrmann A, Beutelmann V, Zwanzger P, Lenzen T, Grotegerd D, et al. Limbic scars: long-term consequences of childhood maltreatment revealed by functional and structural magnetic resonance imaging. Biol Psychiatry. 2012;71:286-93.

9. Kitayama N, Vaccarino V, Kutner M, Weiss P, Bremner JD. Magnetic resonance imaging (MRI) measurement of hippocampal volume in posttraumatic stress disorder: a meta-analysis. J Affect Disord. 2005;88:79-86.

10. Choi J, Jeong B, Rohan ML, Polcari AM, Teicher MH. Preliminary evidence for white matter tract abnormalities in young adults exposed to parental verbal abuse. Biol Psychiatry. 2009;65:227-34.

11. Raine A, Park S, Lencz T, Bihrle $S$, LaCasse $L$, Widom $C S$, et al. Reduced right hemisphere activation in severely abused violent offenders during a working memory task: an fMRI study. Aggress Behav. 2001;27:111-29. 
12. Dillon DG, Holmes AJ, Birk JL, Brooks N, Lyons-Ruth K, Pizzagalli DA. Childhood adversity is associated with left basal ganglia dysfunction during reward anticipation in adulthood. Biol Psychiatry. 2009;66:206-13.

13. Bolhuis JJ, Honey RC. Imprinting, learning and development: from behavior to brain and back. Trends Neurosci. 1998;21:306-11.

14. Weaver IC, Cervoni N, Champagne FA, D'Alessio AC, Sharma S, Seckl JR, et al. Epigenetic programming by maternal behavior. Nat Neurosci. 2004;7:847-54.

15. Fortuin J, van Geel M, Vedder P. Peer influences on internalizing and externalizing problems among adolescents: a longitudinal social network analysis. J Youth Adolesc. 2015;44:887-97.

16. Hart H, Rubia K. Neuroimaging of child abuse: a critical review. Front Hum Neurosci. 2012;6:52.

17. Bick J, Nelson CA. Early adverse experiences and the developing brain. Neuropsychopharmacology . 2016;41:177-96.

18. McCrory E, De Brito SA, Viding E. The impact of childhood maltreatment: a review of neurobiological and genetic factors. Front Psychiatry. 2011;2:1-14.

19. Carrion VG, Weems CF, Watson C, Eliez S, Menon V, Reiss AL. Converging evidence for abnormalities of the prefrontal cortex and evaluation of midsagittal structures in pediatric posttraumatic stress disorder: An MRI study. Psychiatry Res. 2009;172:226-34.

20. De Brito SA, Viding E, Sebastian CL, Kelly PA, Mechelli A, Maris H, et al. Reduced orbitofrontal and temporal grey matter in a community sample of maltreated children. J Child Psychol Psychiatry. 2013;54:105-12.

21. McGowan PO, Sasaki A, D'Alessio AC, Dymov S, Labonté B, Szyf M, et al. Epigenetic regulation of the glucocorticoid receptor in human brain associates with childhood abuse. Nat Neurosci. 2009;12:342-8.

22. Zannas AS, Provençal N, Binder EB. Epigenetics of posttraumatic stress disorder: current evidence, challenges, and future directions. Biol Psychiatry. 2015;78: 327-35.

23. Simons RL, Lei MK, Beach SRH, Cutrona CE, Philibert RA. Methylation of the oxytocin receptor gene mediates the effect of adversity on negative schemas and depression. Dev Psychopathol. 2017;29:725-36.

24. Gouin JP, Zhou QQ, Booij L, Boivin M, Côté SM, Hébert $M$, et al. Associations among oxytocin receptor gene (OXTR) DNA methylation in adulthood, exposure to early life adversity, and childhood trajectories of anxiousness. Sci Rep. 2017;7:7446

25. Smearman EL, Almli LM, Conneely KN, Brody GH, Sales JM, Bradley B, et al. Oxytocin receptor genetic and epigenetic variations: association with child abuse and adult psychiatric symptoms. Child Dev. 2016;87:122-34.

26. Donaldson ZR, Young L. Oxytocin, vasopressin, and the neurogenetics of sociality. Science . 2008;322:900-4.

27. Feldman R, Gordon I, Zagoory-Sharon O. Maternal and paternal plasma, salivary, and urinary oxytocin and parent-infant synchrony: considering stress and affiliation components of human bonding. Dev Sci. 2011;14:752-61.

28. Wismer Fries AB, Ziegler TE, Kurian JR, Jacoris S, Pollak SD. Early experience in humans is associated with changes in neuropeptides critical for regulating social behavior. Proc Natl Acad Sci USA. 2005;102:17237-40.

29. Pierrehumbert B, Torrisi R, Laufer D, Halfon O, Ansermet F, Beck Popovic M. Oxytocin response to an experimental psychosocial challenge in adults exposed to traumatic experiences during childhood or adolescence. Neuroscience. 2010;166:168-77.

30. Sheehan DV, Sheehan KH, Shytle RD, Janavs J, Bannon Y, Rogers JE, et al. Reliability and validity of the Mini International neuropsychiatric interview for children and adolescents (MINI-KID). J Clin Psychiatry. 2010;71:313-26.

31. Wechsler D. Wechsler Intelligence Scale for Children. 4th ed. San Antonio, TX: Psychological Corporation; 2003.

32. Wechsler D. Wechsler Adult Intelligence Scale, 3rd ed. San Antonio, TX: Psychological Corporation; 1997.

33. Sanders B, Becker-Lausen E. The measurement of psychological maltreatment: early data on the Child Abuse and Trauma Scale. Child Abus Negl. 1995;19: 315-23.

34. Birleson P. The validity of depressive disorder in childhood and the development of a self-rating scale: a research report. J Child Psychol Psychiatry. 1981;22:73-88.

35. Achenbach TM. Manual for the child behavior checklist/4-18 and 1991 profile. Burlington, VT: Department of Psychiatry, University of Vermont; 1991.

36. Hazan C, Shaver P. Romantic love conceptualized as an attachment process. J Pers Soc Psychol. 1987;52:511-24.

37. Collins NL, Read SJ. Adult attachment, working models, and relationship quality in dating couples. J Pers Soc Psychol. 1990;58:644-63.

38. Takuma T, Toda K. Interpersonal attitude in adolescence from the viewpoint of attachment theory. J Soc Sci Humanit (Jimbun gakuho). 1988;196:1-16.

39. Kasuya K, Kawamura S. Development of an internal working model for junior high school students: examination of reliability and validity. Jpn J Couns Sci. 2005;38: $141-8$.
40. Kusui C, Kimura T, Ogita K, Nakamura H, Matsumura Y, Koyama M, et al. DNA methylation of the human oxytocin receptor gene promoter regulates tissuespecific gene suppression. Biochem Biophys Res Commun. 2001;289:681-86.

41. Nishitani S, Parets SE, Haas BW, Smith AK. DNA methylation analysis from saliva samples for epidemiological studies. Epigenetics . 2018;13:352-62.

42. BLUEPRINT consortium. Quantitative comparison of DNA methylation assays for biomarker development and clinical applications. Nat Biotechnol. 2016;34: 726-37.

43. Coolen MW, Statham AL, Gardiner-Garden M, Clark SJ. Genomic profiling of CpG methylation and allelic specificity using quantitative high-throughput mass spectrometry: critical evaluation and improvements. Nucleic Acids Res. 2007;35: e119.

44. Koolschijn PC, van Haren NE, Lensvelt-Mulders GJ, Hulshoff Pol HE, Kahn RS. Brain volume abnormalities in major depressive disorder: a meta-analysis of magnetic resonance imaging studies. Hum Brain Mapp. 2009:30:3719-35.

45. Takiguchi S, Fujisawa TX, Mizushima S, Saito DN, Shimada K, Okamoto Y, et al. The neural effects of intranasal oxytocin administration in reactive attachment disorder. No To Hattatsu. 2016;48:S234.

46. Boccia ML, Petrusz $P$, Suzuki K, Marson L, Pedersen CA. Immunohistochemical localization of oxytocin receptors in human brain. Neuroscience. 2013;253: 155-64.

47. Maldjian JA, Laurienti PJ, Kraft RA, Burdette JH. An automated method for neuroanatomic and cytoarchitectonic atlas-based interrogation of $\mathrm{fMRI}$ data sets. Neuroimage . 2003;19:1233-9.

48. Rubin LH, Connelly JJ, Reilly JL, Carter CS, Drogos LL, Pournajafi-Nazarloo H, et al. Sex and diagnosis specific associations between DNA methylation of the oxytocin receptor gene with emotion processing and temporal-limbic and prefrontal brain volumes in psychotic disorders. Biol Psychiatry Cogn Neurosci Neuroimaging. 2016;1:141-51

49. Haas BW, Filkowski MM, Cochran RN, Denison L, Ishak A, Nishitani S, et al. Epigenetic modification of OXT and human sociability. Proc Natl Acad Sci USA. 2016;113:E3816-23.

50. Unternaehrer E, Meyer AH, Burkhardt SC, Dempster E, Staehli S, Theill N, et al. Childhood maternal care is associated with DNA methylation of the genes for brain-derived neurotrophic factor (BDNF) and oxytocin receptor (OXTR) in peripheral blood cells in adult men and women. Stress . 2015;18:451-61.

51. Needham BL, Smith JA, Zhao W, Wang X, Mukherjee B, Kardia SL, et al. Life course socioeconomic status and DNA methylation in genes related to stress reactivity and inflammation: the multi-ethnic study of atherosclerosis. Epigenetics. 2015; 10:958-69.

52. Puglia MH, Lillard TS, Morris JP, Connelly JJ. Epigenetic modification of the oxytocin receptor gene influences the perception of anger and fear in the human brain. Proc Natl Acad Sci USA. 2015;112:3308-13.

53. Perkeybile AM, Carter CS, Wroblewski KL, Puglia MH, Kenkel WM, Lillard TS, et al. Early nurture epigenetically tunes the oxytocin receptor. Psychoneuroendocrinology. 2019;99:128-36.

54. Gregory SG, Connelly JJ, Towers AJ, Johnson J, Biscocho D, Markunas CA, et al. Genomic and epigenetic evidence for oxytocin receptor deficiency in autism. BMC Med. 2009;7:62

55. Yoshida M, Takayanagi $Y$, Inoue K, Kimura T, Young LJ, Onaka T, et al. Evidence that oxytocin exerts anxiolytic effects via oxytocin receptor expressed in serotonergic neurons in mice. J Neurosci. 2009;29:2259-71.

56. Bethlehem RAI, Lombardo MV, Lai MC, Auyeung B, Crockford SK, Deakin J, et al. Intranasal oxytocin enhances intrinsic corticostriatal functional connectivity in women. Transl Psychiatry. 2017;7:e1099.

57. Vrtička $P$, Vuilleumier $P$. Neuroscience of human social interactions and adult attachment style. Front Hum Neurosci. 2012;6:212.

58. Rilling JK, Young LJ. The biology of mammalian parenting and its effect on offspring social development. Science . 2014;345:771-6.

59. Skuse DH, Gallagher L. Dopaminergic-neuropeptide interactions in the social brain. Trends Cogn Sci. 2009;13:27-35.

60. Quintana DS, Rokicki J, van der Meer D, Alnæs D, Kaufmann T, Córdova-Palomera A. Oxytocin pathway gene networks in the human brain. Nat Commun. 2019; 10:668.

61. Xiao L, Priest MF, Nasenbeny J, Lu T, Kozorovitskiy Y. Biased oxytocinergic modulation of midbrain dopamine systems. Neuron. 2017:95:368-84.

62. Feldman R. The Neurobiology of Human Attachments. Trends Cogn Sci. 2017;21: 80-99.

63. Gillath O, Bunge SA, Shaver PR, Wendelken C, Mikulincer M. Attachment style differences in the ability to suppress negative thoughts: exploring the neural correlates. Neuroimage . 2005;28:835-47.

64. Vrtička P, Sander D, Anderson B, Badoud D, Eliez S, Debbané M. Social feedback processing from early to late adolescence: influence of sex, age, and attachment style. Brain Behav. 2014;4:703-20. 
Oxytocin receptor DNA methylation and alterations of brain volumes in... TX. Fujisawa et al.

65. Friston KJ, Rotshtein P, Geng JJ, Sterzer P, Henson RN. A critique of functional localisers. Neuroimage. 2006;30:1077-87.

66. Smith AK, Kilaru V, Klengel T, Mercer KB, Bradley B, Conneely KN, et al. DNA extracted from saliva for methylation studies of psychiatric traits: evidence tissue specificity and relatedness to brain. Am J Med Genet B Neuropsychiatr Genet. 2015;168B:36-44.

67. Braun PR, Han S, Hing B, Nagahama Y, Gaul LN, Heinzman JT, et al. Genome-wide DNA methylation comparison between live human brain and peripheral tissues within individuals. Transl Psychiatry. 2019;9:47.
68. Theda C, Hwang SH, Czajko A, Loke YJ, Leong P, Craig JM. Quantitation of the cellular content of saliva and buccal swab samples. Sci Rep. 2018;8:6944.

69. Smyke AT, et al. A randomized controlled trial comparing foster care and institutional care for children with signs of reactive attachment disorder. Am J Psychiatry. 2012;169:508-14.

70. De Bellis MD, Woolley DP, Hooper SR. Neuropsychological findings in pediatric maltreatment: relationship of PTSD, dissociative symptoms, and abuse/neglect indices to neurocognitive outcomes. Child Maltreat. 2013;18: $171-83$. 